TITLE:

\title{
Space-Time Grains: Roots of Special and Doubly Special Relativity
}

\author{
$\operatorname{AUTHOR}(\mathrm{S}):$ \\ Jizba, Petr; Scardigli, Fabio
}

CITATION:

Jizba, Petr ... [et al]. Space-Time Grains: Roots of Special and Doubly Special Relativity. Foundations of Physics 2014, 44(5): 512-522

ISSUE DATE:

2014-05

URL:

http://hdl.handle.net/2433/199608

\section{RIGHT:}

The final publication is available at Springer via http://dx.doi.org/10.1007/s10701-0139758-9.; この論文は出版社版でありません。引用の際には出版社版をご確認ご利用くだ さい。; This is not the published version. Please cite only the published version. 
Noname manuscript No.

(will be inserted by the editor)

Petr Jizba - Fabio Scardigli

\title{
Space-time grains: roots of special and doubly special Relativity
}

Received: date / Accepted: date

\begin{abstract}
We show that the special relativistic dynamics when combined with quantum mechanics and the concept of superstatistics can be interpreted as arising from two interlocked non-relativistic stochastic processes that operate at different energy scales. This interpretation leads to Feynman amplitudes that are in the Euclidean regime identical to transition probability of a Brownian particle propagating through a granular space. Some kind of spacetime granularity could be therefore held responsible for the emergence at larger scales of various symmetries. For illustration we consider also the dynamics and the propagator of a spinless relativistic particle. Implications for doubly special relativity, quantum field theory, quantum gravity and cosmology are discussed.
\end{abstract}

\section{Introduction}

The concept of "emergence" plays an important role in quantum field theory and, in particular, in particle and condensed matter physics, since it embodies the essential feature of systems with several interlocked time scales. In these systems, the observed macroscopic-scale dynamics and related degrees of freedom differ drastically from the actual underlying microscopic-scale physics [1]. Superstatistics provides a specific realization of this paradigm:

Petr Jizba

FNSPE, Czech Technical University in Prague,

Břehová 7, 11519 Praha 1, Czech Republic

E-mail: p.jizba@fjfi.cvut.cz

Fabio Scardigli (*corresponding author)

Dipartimento di Matematica, Politecnico di Milano,

Piazza Leonardo da Vinci 32, 20133 Milano, Italy

and Yukawa Institute for Theoretical Physics, Kyoto University,

Kyoto 606-8502, Japan

E-mail: fabio@phys.ntu.edu.tw 
It predicts that the emergent behavior can be often regarded as a superposition of several statistical systems that operate at different spatio-temporal scales $[2,3]$. In particular, many applications have recently been reported, in hydrodynamic turbulence [4], turbulence in quantum liquids [5], pattern forming systems [6] or scattering processes in high-energy physics [7].

The essential assumption of the superstatistics scenario is the existence of sufficient spatio-temporal scale separations between relevant dynamics within the studied system so that the system has enough time to relax to a local equilibrium state and to stay within it for some time. In practical applications one is typically concerned with two scales. Following Ref. [2], we consider an intensive parameter $\zeta$ that fluctuates on a much larger time scale than the typical relaxation time of the local dynamics. The random variable $\zeta$ can be in practice identified, e.g., with the inverse temperature [2,3], friction constant [8], volatility [9] or einbein [10]. On intuitive ground, one may understand the superstatistics by using the adiabatic Ansatz. Namely, the system under consideration, during its evolution, travels within its state space $X$ (described by state variable $A \in X$ ) which is partitioned into small cells characterized by a sharp value of $\zeta$. Within each cell, the system is described by the conditional distribution $p(A \mid \zeta)$. As $\zeta$ varies adiabatically from cell to cell, the joint distribution of finding the system with a sharp value of $\zeta$ in the state $A$ is $p(A, \zeta)=p(A \mid \zeta) p(\zeta)$ (Bayes theorem). The resulting macroscale (emergent) statistics $p(A)$ for finding system in the state $A$ is obtain by eliminating the nuisance parameter $\zeta$ through marginalization, that is

$$
p(A)=\int p(A \mid \zeta) p(\zeta) d \zeta .
$$

Interestingly enough, the sufficient time scale separation between two relevant dynamics in a studied system allows to qualify superstatistics as a form of slow modulation [11].

In this paper, we recast the Feynman transition amplitude of a relativistic scalar particle into a form, which (after being analytically continued to imaginary times) coincides with a superstatistics marginal probability (1). The derivation is based on the Lévy-Khinchine theorem for infinitely divisible distributions [12,13], and for illustration we consider the dynamics and the propagator of a Klein-Gordon (i.e., neutral spin-0) particle. Our reasonings can be also extended to charged spin -0 , spin $-\frac{1}{2}$, Proca's spin -1 particles and to higher-spin particles phrased via the Bargmann-Wigner wave equation [10]. Further generalization to external electromagnetic potential has been reported in Refs. [10,14].

We also argue that the above formulation can be looked at as if the particle would randomly propagate (in the sense of Brownian motion) through an inhomogeneous or granular medium ("vacuum") [14].

Our argument is based upon a recent observation $[9,10,14]$ that the Euclidean path integral (PI) for relativistic particles may be interpreted as describing a doubly-stochastic process that operates at two separate spatiotemporal scales. The short spatial scale, which is much smaller than particle's Compton length $\lambda_{C}=1 / m c(\hbar=1)$, describes a Wiener (i.e., Galilean relativity) process with a sharp (Galilean-invariant) Newtonian mass. The large 
spatial scale, which is of order $\lambda_{C}$, corresponds to distances over which the fluctuating Newtonian mass changes appreciably. At scales much larger than $\lambda_{C}$ the particle evolves according to a genuine relativistic dynamics, with a sharp value of the mass coinciding with the Einstein rest mass. Particularly striking is the fact that when we average the particle's velocity over the structural correlation distance (i.e., over particle's $\lambda_{C}$ ) we obtain the velocity of light $c$. So the picture that emerges from this analysis is that the particle (with a non-zero mass!) propagates over the correlation distance $\lambda_{C}$ with an average velocity $c$, while at larger distance scales (i.e., when a more coarse grained view is taken) the particle propagates as a relativistic particle with a sharp mass and an average velocity that is subluminal. Quite remarkably, one can observe an identical behavior in the well-known Feynman's checkerboard PI $[16,17]$ to which the transition amplitude (1) reduces in the case of a relativistic Dirac fermion in $1+1$ dimensions $[10,14]$.

A considerably expanded presentation including the issue of reparametrization invariance, bibliography, and proofs of the main statements and formulas has been given in a companion paper [14].

\section{Superstatistics path integrals}

When a conditional probability density function (PDF) is formulated through a PI, then it satisfies the Einstein-Smoluchowski equation (ESE) for continuous Markovian processes, namely [15]

$$
p\left(y, t^{\prime \prime} \mid x, t\right)=\int_{-\infty}^{\infty} \mathrm{d} z p\left(y, t^{\prime \prime} \mid z, t^{\prime}\right) p\left(z, t^{\prime} \mid x, t\right),
$$

with $t^{\prime}$ being any time between $t^{\prime \prime}$ and $t$. Conversely, any transition probability satisfying ESE possesses a PI representation [16]. In physics one often encounters probabilities formulated as a superposition of PI's,

$$
\begin{aligned}
& \wp\left(x^{\prime}, t^{\prime} \mid x, t\right) \\
& =\int_{0}^{\infty} \mathrm{d} \zeta \omega(\zeta, T) \int_{x(t)=x}^{x\left(t^{\prime}\right)=x^{\prime}}[\mathrm{d} x \mathrm{~d} p] e^{\int_{t}^{t^{\prime}} \mathrm{d} \tau(\mathrm{i} p \dot{x}-\zeta H(p, x))} .
\end{aligned}
$$

Here $\omega(\zeta, T)$ with $T=t^{\prime}-t$ is a normalized PDF defined on $\mathbb{R}^{+} \times \mathbb{R}^{+}$. The form (3) typically appears in non-perturbative approximations to statistical partition functions, in polymer physics, in financial markets, in systems with reparametrization invariance, etc. The random variable $\zeta$ is then related to the inverse temperature, coupling constant, volatility, vielbein, etc.

The existence of different time scales and the flow of the information from slow to fast degrees of freedom typically creates the irreversibility on the macroscopical level of the description. The corresponding information thus is not lost, but passes in a form incompatible with the Markovian description. The most general class of distributions $\omega(\zeta, T)$ on $\mathbb{R}^{+} \times \mathbb{R}^{+}$for which the superposition of Markovian processes remain Markovian, i.e., when also $\wp\left(x^{\prime}, t^{\prime} \mid x, t\right)$ satisfies the ESE (2), was found in Ref. [9]. The key is to note that 
in order to have (2) satisfied by $\wp$, the rescaled PDF $w(\zeta, T) \equiv \omega(\zeta / T, T) / T$ should satisfy the ESE for homogeneous Markovian process

$$
w\left(\zeta, t_{1}+t_{2}\right)=\int_{0}^{\zeta} \mathrm{d} \zeta^{\prime} w\left(\zeta^{\prime}, t_{1}\right) w\left(\zeta-\zeta^{\prime}, t_{2}\right) .
$$

Consequently the Laplace image fulfills the functional equation with $t_{1}, t_{2} \in$ $\mathbb{R}^{+}$. By assuming continuity in $T$, it follows that the multiplicative semigroup $\tilde{w}\left(p_{\zeta}, T\right)_{T \geq 0}$ satisfies $\tilde{w}\left(p_{\zeta}, T\right)=\left\{\tilde{w}\left(p_{\zeta}, 1\right)\right\}^{T}$. From this we see that the distribution of $\zeta$ at $T$ is completely determined by the distribution of $\zeta$ at $T=1$. In addition, because $\tilde{w}\left(p_{\zeta}, 1\right)=\left\{\tilde{w}\left(p_{\zeta}, 1 / n\right)\right\}^{n}$ for any $n \in \mathbb{N}^{+}, w(\zeta, 1)$ is infinitely divisible. The Lévy-Khinchine theorem $[12,13]$ then ensures that $\log \tilde{w}\left(p_{\zeta}, T\right) \equiv-T F\left(p_{\zeta}\right)$ must have the generic form

$$
\log \tilde{w}\left(p_{\zeta}, T\right)=-T\left(\alpha p_{\zeta}+\int_{0}^{\infty}\left(1-e^{-p_{\zeta} u}\right) \nu(\mathrm{d} u)\right),
$$

where $\alpha \geq 0$ is a drift constant and $\nu$ is some non-negative measure on $(0, \infty)$ satisfying $\int_{\mathbb{R}^{+}} \min (1, u) \nu(\mathrm{d} u)<\infty$. Finally the Laplace inverse of $\tilde{w}\left(p_{\zeta}, T\right)$ yields $\omega(\zeta, T)$. Once $\omega(\zeta, T)$ is found, then $\wp\left(x^{\prime}, t^{\prime} \mid x, t\right)$ possesses a PI representation on its own. What is the form of the new Hamiltonian? To this end we rewrite (3) in Dirac operator form as [9]

$$
\begin{aligned}
& \wp\left(x^{\prime}, t^{\prime} \mid x, t\right)=\left\langle x^{\prime}\left|\int_{0}^{\infty} \mathrm{d} \zeta w(\zeta, T) e^{-\zeta \hat{H}}\right| x\right\rangle \\
& =\left\langle x^{\prime}\left|\{\tilde{w}(\hat{H}, 1)\}^{T}\right| x\right\rangle=\left\langle x^{\prime}\left|e^{-T F(\hat{H})}\right| x\right\rangle .
\end{aligned}
$$

Hence, the identification $\mathcal{H}(\boldsymbol{p}, \boldsymbol{x})=F(H(\boldsymbol{p}, \boldsymbol{x}))$ can be made. Here one might worry about the operator-ordering problem. For our purpose it suffices to note that when $H$ is $\boldsymbol{x}$-independent, the former relation is exact. In more general instances the Weyl ordering is a natural choice because in this case the required mid-point rule follows automatically and one does not need to invoke the gauge invariance $[9,29]$. In situations when other non-trivial configuration space symmetries (such as non-holonomic symmetry) are required, other orderings might be more physical [9].

\section{Statistical origin of Special Relativity - Granular Universe}

The Feynman transition amplitudes (or better its Euclidean version - transition probabilities) naturally fits into the structure of superstatistics PI's discussed above.

Note first that the choice $\alpha=0$ and $\nu(\mathrm{d} u)=1 /\left(2 \sqrt{\pi} u^{3 / 2}\right) \mathrm{d} u$ leads to $F\left(p_{\zeta}\right)=\sqrt{p_{\zeta}}$. This identifies $w(\zeta, T)$ with the (unshifted) Lévy distribution with the scale parameter $T^{2} / 2$. Moreover, when $H(\boldsymbol{p}, \boldsymbol{x})=\boldsymbol{p}^{2} c^{2}+m^{2} c^{4}$ then 
(3) can be cast into the form (see also Refs. $[9,10,14]$ )

$$
\begin{aligned}
& \int_{\boldsymbol{x}(0)=\boldsymbol{x}}^{\boldsymbol{x}(T)=\boldsymbol{x}^{\prime}}[\mathrm{d} \boldsymbol{x} \mathrm{d} \boldsymbol{p}] \exp \left\{\int_{0}^{T} \mathrm{~d} \tau\left[\mathrm{i} \boldsymbol{p} \cdot \dot{\boldsymbol{x}}-c \sqrt{\boldsymbol{p}^{2}+m^{2} c^{2}}\right]\right\} \\
& =\int_{0}^{\infty} \mathrm{dm}_{\frac{1}{2}}\left(\mathfrak{m}, T c^{2}, T c^{2} m^{2}\right) \int_{\boldsymbol{x}(0)=\boldsymbol{x}}^{\boldsymbol{x}(T)=\boldsymbol{x}^{\prime}}[\mathrm{d} \boldsymbol{x} \mathrm{d} \boldsymbol{p}] \exp \left\{\int_{0}^{T} \mathrm{~d} \tau\left[\mathrm{i} \boldsymbol{p} \cdot \dot{\boldsymbol{x}}-\frac{\boldsymbol{p}^{2}}{2 \mathfrak{m}}-m c^{2}\right]\right\},
\end{aligned}
$$

where $t^{\prime}-t=T$, and

$$
\mathrm{f}_{p}(z, a, b)=\frac{(a / b)^{p / 2}}{2 K_{p}(\sqrt{a b})} z^{p-1} \mathrm{e}^{-(a z+b / z) / 2},
$$

is the generalized inverse Gaussian distribution [13] $\left(K_{p}\right.$ is the modified Bessel function of the second kind with index $p$ ). The LHS of (8) represents the PI for the free spinless relativistic particle in the Newton-Wigner representation [18]. The full Klein-Gordon (KG) kernel which also contains the negative-energy spectrum can be obtained from (8) by considering the Feshbach-Villars representation of the KG equation and making the substitution [10]

$$
\mathrm{f}_{\frac{1}{2}}\left(\mathfrak{m}, t c^{2}, t c^{2} m^{2}\right) \mapsto \frac{1+\operatorname{sgn}(t) \sigma_{3}}{2} \mathrm{f}_{\frac{1}{2}}\left(\mathfrak{m},|t| c^{2},|t| c^{2} m^{2}\right) .
$$

The matrix nature of the smearing distribution $\left(\sigma_{3}\right.$ is the Pauli matrix) naturally includes the Feynman-Stuckelberg causal boundary condition and thus treats both particles and antiparticles in a symmetric way $[10,19]$. When the partition function is going to be calculated, the trace will get rid of the $\operatorname{sgn}(t)$ term and $1 / 2$ is turned to 1 .

The explicit form of the identity (8) indicates that $\mathfrak{m}$ can be interpreted as a Galilean-invariant Newton-like mass which takes on continuous values distributed according to $\mathrm{f}_{\frac{1}{2}}\left(\mathfrak{m}, T c^{2}, T c^{2} m^{2}\right)$ with $\langle\tilde{m}\rangle=m+1 / T c^{2}$ and $\operatorname{var}(\mathfrak{m})=m / T c^{2}+2 / T^{2} c^{4}$. Fluctuations of the Newtonian mass can be then depicted as originating from particle's evolution in an inhomogeneous or granular medium. Granularity, as known, for example, from solid-state systems, typically leads to corrections in the local dispersion relation [20] and hence to alterations in the local effective mass. The following picture thus emerges: on the short-distance scale, a non-relativistic particle can be envisaged as propagating via classical Brownian motion through a single grain with a local mass $\mathfrak{m}$. This fast-time process has a time scale $\sim 1 / \mathfrak{m} c^{2}$. An averaged value of the local time scale represents a transient temporal scale $\left\langle 1 / \mathfrak{m} c^{2}\right\rangle=1 / m c^{2}$ which coincides with particle's Compton time $T_{C}$ - i.e., the time for light to cross the particle's Compton wavelength. At time scales much longer than $T_{C}$ (large-distance scale), the probability that the particle encounters a grain which endows it with a mass $\mathfrak{m}$ is $\mathrm{f}_{\frac{1}{2}}\left(\mathfrak{m}, T c^{2}, T c^{2} m^{2}\right)$. As a result one may view a single-particle relativistic theory as a single-particle non-relativistic theory where the particle's Newtonian mass $\mathfrak{m}$ represents a fluctuating parameter which approaches on average the Einstein rest mass $m$ in the large $t$ 
limit. We stress that $t$ should be understood as the observation time, a time after which the observation (position measurement) is made. In particular, during the period $t$ the system remains unperturbed. One can thus justly expect that in the long run all mass fluctuations will be washed out and only a sharp time-independent effective mass will be perceived. The form of $\langle\mathfrak{m}\rangle$ identifies the time scale at which this happens with $t \sim 1 / m c^{2}$, i.e. with the Compton time $T_{C}$. It should be stressed that above mass fluctuations have nothing to do with the Zitterbewegung which is caused by interference between positive- and negative-energy wave components. In our formulation both regimes are decoupled.

We may also observe that by coarse-graining the velocity over the correlation time $T_{C}$ we have

$$
\langle|\boldsymbol{v}|\rangle_{T_{C}}=\left.\frac{\langle|\boldsymbol{p}|\rangle}{\langle\mathfrak{m}\rangle}\right|_{T_{C}}=c
$$

So on a short-time scale of order $\lambda_{C}$ the spinless relativistic particle propagates with an averaged velocity which is the speed of light $c$. But if one checks the particle's position at widely separated intervals (much larger than $\lambda_{C}$ ), then many directional reversals along a typical PI trajectory will take place, and the particle's net velocity will be then less than $c$ - as it should be for a massive particle. The particle then acquires a sharp mass equal to Einstein's mass, and the process (not being hindered by fluctuating masses) is purely Brownian. This conclusion is in line with the well-known Feynman checkerboard picture $[14,17]$ to which it reduces in the case of $(1+1) \mathrm{D}$ relativistic Dirac particle.

\section{Emergent doubly special relativity}

Understanding the robustness of the emergent Special Relativity under small variations in the mass-smearing distribution function $\mathrm{f}_{\frac{1}{2}}$ can guide the study of the relation between Einsteinian SR and other deformed variants of SR, such as Magueijo-Smolin and Amelino-Camelia's doubly special relativity [21, $22]$, or (quantum) $\kappa$-Poincaré deformation of relativistic kinematics [23]. In DRS models a further invariant scale $\ell$ is introduced, besides the usual speed of light $c$, and $\ell$ is typically considered to be of order of the Planck length. A small variation $\delta \mathrm{f}_{\frac{1}{2}}$ of the smearing function originates the new Hamiltonian

$$
\bar{H}=\frac{\epsilon_{1}}{4}+\left(1+\frac{\epsilon_{0}}{2}\right) \sqrt{\boldsymbol{p}^{2} c^{2}+m^{2} c^{4}+\frac{\epsilon_{2}}{4}},
$$

with $\epsilon_{1}=-2\left(1+\epsilon_{0} / 2\right) \sqrt{\epsilon_{2}}$ (see Ref. [14] for details). By setting

$$
\epsilon_{1}=2\left(\sqrt{\frac{1}{1-c^{2} m^{2} \ell^{2}}}-1\right), \quad \epsilon_{2}=\frac{4 c^{6} m^{4} \ell^{2}}{1-c^{2} m^{2} \ell^{2}},
$$


the new Hamiltonian $\bar{H}$ can be easily identified with

$$
\bar{H}=c \frac{-m^{2} c^{2} \ell \mp \sqrt{\boldsymbol{p}^{2}\left(1-m^{2} c^{2} \ell^{2}\right)+m^{2} c^{2}}}{1-m^{2} c^{2} \ell^{2}},
$$

which coincides with the Magueijo-Smolin's doubly special relativistic Hamiltonian, in, say, its version [24]. It should be stressed that the Hamiltonian (11) (when also negative energy states are included) violates CPT symmetry. This is a typical byproduct of the Lorentz symmetry violation in many deformed SR systems.

For the Hamiltonian (12) a relation analog to (8) holds, where now the smearing function has the form $\mathrm{f}_{\frac{1}{2}}\left(\mathfrak{m}, T c^{2} \lambda, T c^{2} m^{2} \lambda\right)$ with $\lambda=1 /(1-$ $\left.m^{2} c^{2} \ell^{2}\right)$. The correlation distance $1 / m c \lambda$ can be naturally assumed as the minimal size $L_{\text {GRAIN }}$ of the "grain of space" of the polycrystalline medium, which is linked to the new invariant scale $\ell$ by

$$
L_{\text {GRAIN }}:=\frac{1}{m c \lambda}=\lambda_{C}\left(1-m^{2} c^{2} \ell^{2}\right) .
$$

By tuning the size $L_{\text {GRAIN }}$ of these "grains of space" it is possible to pass continuously from Lorenz symmetry to other different symmetries, as those enjoyed by DSR models. We can in principle speculate that each large scale symmetry could originate from a specific kind of space(time) granularity.

\section{Quantum field theory}

The superstatistics transition probability (6) was constructed on the premise that $H$ is associated with a single particle. Of course, a single-particle relativistic quantum theory is logically untenable, since a multi-particle production is allowed whenever the particle reaches the threshold energy for pair production. In addition, Leutwyler's no-interaction theorem [25] prohibits interaction for any finite number of particles in the context of relativistic mechanics. To evade the no-interaction theorem it is necessary to have an infinite number of degrees of freedom to describe interaction. The latter is typically achieved via local quantum field theories (QFT).

It should be underlined in this context that the PI for a single relativistic particle is still a perfectly legitimate building block even in QFT. Recall that in the standard perturbative treatment of, say, generating functional for a scalar field each Feynman diagram is composed of integrals over product of free correlation functions (Feynman's correlators):

$$
\Delta_{F}\left(\boldsymbol{y}, c t_{y} ; \boldsymbol{z}, c t_{z}\right)=\frac{1}{4} \int_{-\infty}^{\infty} \mathrm{d} \tau \operatorname{sgn}\left(\tau-t_{y}\right) \wp\left(\boldsymbol{y}, \tau \mid \boldsymbol{z}, t_{z}\right),
$$

and may thus be considered as a functional of the PI $\wp\left(\mathbf{x}^{\prime}, t^{\prime} \mid \mathbf{x}, t\right)$. In fact, QFT in general, can be viewed as a grand-canonical ensemble of fluctuating particle histories (worldlines) where Feynman diagrammatic representation of quantum fields depicts directly the pictures of the world-lines in a grandcanonical ensemble. This is the so-called "worldline quantization" of particle 
physics, and is epitomized, e.g., in Feynman's worldline representation of the one-loop affective action in quantum electrodynamics [26], in Kleinert's disorder field theory [27] or in the Bern-Kosower and Strassler "string-inspired" approaches to QFT [28].

Because of (14), the relationship between bosonic Bern-Kosower Green's function $G_{B}\left(\tau_{1}, \tau_{2}\right)$ and the PI $\wp\left(\mathbf{x}^{\prime}, t^{\prime} \mid \mathbf{x}, t\right)$ can be found easily through the known functional relation between $G_{B}$ and $\Delta_{F}$, cf. Refs. [28].

\section{Gravity, local Lorentz invariance, and Cosmology}

When spacetime is curved, a metric tensor enters in both PI's in (7) in a different way, yielding different "counterterms" [15, 29]. For instance, in Bastianelli-van Nieuwenhuizen's time slicing regularization scheme [29] one has (when $\hbar$ is reintroduced)

$$
\begin{aligned}
& \frac{\boldsymbol{p}^{2}}{2 \mathfrak{m}} \mapsto \frac{g^{i j} p_{i} p_{j}}{2 \mathfrak{m}}+\frac{\hbar^{2}}{8 \mathfrak{m}}\left(R+g^{i j} \Gamma_{i l}^{m} \Gamma_{j m}^{l}\right), \\
& \sqrt{\boldsymbol{p}^{2}+m^{2} c^{2}} \mapsto \sqrt{g^{i j} p_{i} p_{j}+\frac{\hbar^{2}}{4}\left(R+g^{i j} \Gamma_{i l}^{m} \Gamma_{j m}^{l}\right)+m^{2} c^{2}} \\
& +\hbar^{4} \Phi\left(R, \partial R, \partial^{2} R\right)+\mathcal{O}\left(\hbar^{6}\right),
\end{aligned}
$$

where $g^{i j}, R, \Gamma_{k l}^{j}$ and $\Phi(\ldots)$ are the (space-like) pull-back metric tensor, the scalar curvature, the Christoffel symbol, and non-vanishing function of $R$ and its first and second derivatives, respectively. This causes the superstatistics identity (7) to break down, as can be explicitly checked to the lowest order in $\hbar$. The respective two cases will thus lead to different physics. Because the Einstein equivalence principle requires that the local spacetime structure can be identified with the Minkowski spacetime possessing Lorentz symmetry, one might assume the validity of (8) at least locally. However, in different space-time points one has, in general, a different typical length scale of the local inertial frames, depending on the gravitational field. The characteristic size of the local inertial (i.e. Minkowski) frame is of order $1 /|K|^{1 / 4}$ where $K=R_{\alpha \beta \gamma \delta} R^{\alpha \beta \gamma \delta}$ is the Kretschmann invariant and $R_{\alpha \beta \gamma \delta}$ is the Riemann curvature. Relation (8) tells us that the special relativistic description breaks down in regions of size smaller than $\lambda_{C}$. For curvatures large enough, namely for strong gravitational fields, the size of the local inertial frame can become smaller than $\lambda_{C}$, that is $1 /|K|^{1 / 4} \lesssim \lambda_{C}$. In such regions the special relativistic description is no more valid, and according to (8) must be replaced by a Newtonian description of the events. For instance, in Schwarzschild geometry we have $K=12 r_{s}^{2} / r^{6}$, and the breakdown should be expected at radial distances $r \lesssim\left(\lambda_{C}^{2} r_{s}\right)^{1 / 3}\left(r_{s}\right.$ is the Schwarzschild radius) which are - apart from the hypothetical case of micro-black holes (where $\lambda_{C} \simeq r_{s}$ ) - always deeply buried below the Schwarzschild event horizon. In the cosmologically relevant Friedmann-Lemaître-Robertson-Walker (FLRW) geometry, we have $K=12\left(\dot{a}^{4}+a^{2} \ddot{a}^{2}\right) /(a c)^{4}$, and the breakdown happens when $\left(\dot{a}^{4}+a^{2} \ddot{a}^{2}\right) \gtrsim$ $\left(a c / \lambda_{C}\right)^{4}$, where $a(t)$ is the FLRW scale factor of the Universe and $\dot{a} \stackrel{\gtrsim}{=}$ $\mathrm{d} a / \mathrm{d} t$. Applying the well-known Vilenkin-Ford model [30] for inflationary 
cosmology, where $a(t)$ is given by: $a(t)=A \sqrt{\sinh (B t)}$ with $B=2 c \sqrt{\Lambda / 3}(\Lambda$ is the cosmological constant), we obtain a temporal bound on the validity of local Lorentz invariance, which, expressed in FLRW time, is

$$
t \lesssim \frac{1}{B} \operatorname{arcsinh}\left[\frac{B \lambda_{C}}{\left(8 c^{4}-\left(B \lambda_{C}\right)^{4}\right)^{1 / 4}}\right] \equiv \bar{t} .
$$

By using the presently known [31] value $\Lambda \simeq 10^{-52} \mathrm{~m}^{-2}$ and the $\tau$-lepton Compton's wavelength $\lambda_{C}^{\tau} \simeq 6.7 \times 10^{-16} \mathrm{~m}$ (yielding the tightest upper bound on $t$ ), we obtain $\bar{t} \simeq 4 \times 10^{-24}$ s. Note that, since $B \lambda_{C} \ll c$, then $\bar{t} \simeq \lambda_{C} / c=$ $t_{C}$. Such a violation of the local Lorentz invariance naturally breaks the particle-antiparticle symmetry since there is no unified theory of particles and antiparticles in the non-relativistic physics - formally one has two separate theories. If the resulting matter-antimatter asymmetry provides a large enough CP asymmetry then this might have essential consequences in the early Universe, e.g., for leptogenesis. In this respect, $\bar{t}$ is compatible with the nonthermal leptogenesis period that typically dates between $10^{-26}-10^{-12} \mathrm{~s}$ after the Big Bang.

\section{Conclusions and perspectives}

The new superstatistics PI representation of a relativistic point particle introduced in this Letter, realizes an explicit quantum mechanical duality between Einsteinian and Galilean relativity. It also makes explicit how the SR invariance is encoded in the grain smearing distribution. Notably, the exact LS of a spacetime has no fundamental significance in our analysis, as it is only an accidental symmetry of the coarse-grained configuration space in which a particle executes a standard Wiener process. In passage from grain to grain particles's Newtonian mass fluctuates according to an inverse Gaussian distribution. The observed inertial mass of the particle is thus not a fundamental constant, but it reflects the particle's interaction with the granular vacuum (cosmic field). This, in a sense, supports Mach's view of the phenomenon of inertia.

Interactions can be included in our framework in two different ways. The interaction with a background field (such as electromagnetic field) can be directly treated with the superstatistics prescription (8), see [10]. On the other hand, the multi-particle interactions can be consistently formulated by "embedding" the relativistic PI in QFT via the worldline quantization. Such an embedding may help to study several cosmological implications of systems with granular space. If any of such systems quickly flows to the infrared fixed point, any direct effect due to the space discreteness, and related SR violation, might be insignificant on cosmological scales (where Lorentz and diffeomorphism invariance are restored), while it might be crucial in the early Universe, e.g., for leptogenesis and the ensuing baryogenesis. Consequences on the detailed structure of the Cosmic Microwave Background spectrum will be explored in future work.

The presented approach implies a preferred frame. In this connection it is worth of noting that, despite the fact that (8) is not manifestly LS invariant, 
one may use the Stückelberg trick and introduce a new fictitious variable into the PI (8), in such a way that the new action will have the reparametrization symmetry, but will still be dynamically equivalent to the original action. For relevant details see Ref. [14]. By not knowing the source, one may then view this artificial gauge invariance as being a fundamental or even a defining property of the relativistic theory. One might, however, equally well, proclaim the "polycrystalline" picture as being a basic (or primitive) edifice of SR and view the reparametrization symmetry as a mere artefact of an artificial redundancy that is allowed in our description. It is this second view that we favored here.

The presented scenario cannot directly accommodate the massless particles such as photons (identity (8) holds true only for $m \neq 0$ ). One possibility would be to use the PI representation of Polyakov-Wheeler for massless particles and try to construct a similar superstatistics duality as in the case of massive particles. This procedure is, however, not without technical difficulties and currently is under investigation. Conceptually is far more simpler to assume that the photon has a small mass. At present, there are a number of experimental limits to the mass of the photons [32]. For instance, tests based on Coulomb's law and the galactic vector potential set the upper limit of $m_{\gamma} \lesssim 10^{-18} \mathrm{eV} / \mathrm{c}^{2} \simeq 10^{-57} \mathrm{~g}$. This gives the domain correlation distance for the photon $\simeq 1 / m_{\gamma} c^{2} \simeq 10^{43} \mathrm{~m}$ which is bigger than the radius of observable Universe $\left(\simeq 10^{26} \mathrm{~m}\right)$ and so in this picture the photon mass does not fluctuate - it is a quasi-invariant.

Finally, this approach should reinforce the links between superstatistics paradigm and the approach to quantum gravity based on stochastic quantization [33]. In particular, the outlined granular space could be a natural model for the noise terms in a Parisi-Wu stochastic-like quantization approach to gravity.

\section{Acknowledgement}

The authors are grateful to H. Kleinert, Z. Haba, M. Sakellariadou, and L.S. Schulman for useful feedbacks.

\section{References}

1. see, e.g., P. Anderson, Science 177, 393 (1972); R.B. Laughlin, A Different Universe: Reinventing Physics from the Bottom Down, (Basic Books, London, 2005); I. Licata and A. Sakaji, Physics of Emergence and Organization, (WS, London, 2008)).

2. C. Beck, Phys. Rev. Lett. 87, 180601 (2001); C. Beck and E.G.D. Cohen, Physica A322, 267 (2003); G. Wilk and Z. Wlodarczyk, Phys. Rev. Lett. 85, $2770(2000)$.

3. R. Hanel, S. Thurner and M. Gell-Mann, Generalized entropies and the transformation group of superstatistics, Proceedings of the National Academy of Sciences 108, 6390 (2011).

4. A. Reynolds, Phys. Rev. Lett. 91, 084503 (2003).

5. C. Beck and S. Miah, [arXiv:1207.4062].

6. K.E. Daniels, C. Beck and E. Bodenschatz, Physica D 193, 208 (2004). 
7. G. Wilk and Z. Wlodarczyk, Eur. Phys. J. A 40, 299 (2009); C. Beck, Eur. Phys. J. A 40, 267 (2009).

8. C. Beck, in G. Radons et al.(eds): Anomalous Transport: Foundations and Applications, (John Wiley, London, 2007).

9. P. Jizba and H. Kleinert, Phys. Rev. E 78, 031122 (2008).

10. P. Jizba and H. Kleinert, Phys. Rev. D 82, 085016 (2010).

11. P. Allegrini, F. Barbi, P. Grigolini and P. Paradisi, Phys. Rev. E 73, 046136 (2006).

12. J. Bertoin, Lévy Processes, (CUP, Cambridge, 1996).

13. W. Feller, An Introduction to Probability Theory and its Applications, Vol. II (John Wiley, London, 1966).

14. P. Jizba and F.Scardigli, Phys. Rev. D 86, 025029 (2012).

15. H. Kleinert, Path Integrals in Quantum Mechanics, Statistics, Polymer Physics and Financial Markets (WS, Singapore 2009).

16. R.P. Feynman and A.R. Hibbs, Quantum Mechanics and Path Integrals (McGraw-Hill, New York, 1965).

17. B. Gaveau, T. Jacobson, M. Kac and L.S. Schulman, Phys. Rev. Lett. 53, 419 (1984); T. Jacobson and L.S. Schulman, J. Phys. A 17, 375 (1984).

18. T. Newton and E. Wigner, Rev. Mod. Phys. 21, 400 (1949).

19. H. Feshbach and F. Villars, Rev. Mod. Phys 30, 24 (1958).

20. see, e.g., E.A. Johnson and A. MacKinnon, J. Phys.: Condens. Matter 5 (1993) 5859 .

21. G. Amelino-Camelia, Int. J. Mod. Phys. D 11, 35 (2002); J. Magueijo and L. Smolin, Phys. Rev. Lett. 88, 190403 (2002); G. Amelino-Camelia, Nature 418, 34 (2002).

22. J. Kowalski-Glikman, Introduction to Doubly Special Relativity, Lecture Notes in Physics 669, Springer (2005).

23. M. Daszkiewicz, J. Lukierski and M. Woronowicz, Phys. Rev. D 77, 105007 (2008).

24. J. Magueijo, L. Smolin, Phys. Rev. D 67, 044017 (2003).

25. H. Leutwyler, Nuovo Cimento 37, 556 (1965); G. Marmo, N. Mukunda and E.C.G. Sudarshan, Phys. Rev. D 30, 2110 (1984).

26. R.P. Feynman, Phys. Rev. 80, 440 (1950); Phys. Rev. 84, 108 (1951)

27. H. Kleinert, Gauge Fields in Condensed Matter, Vol. I Superflow and Vortex Lines, (WS, Singapore, 1989).

28. Z. Bern and D.A. Kosower, Phys. Rev. Lett. 66, 1669 (1991); Nucl. Phys. B 379, 451 (1992); M.J. Strassler, Nucl. Phys. B 385, 145 (1992); C. Schubert, Phys. Rep. 335, 73 (2001).

29. F. Bastianelli, P. van Nieuwenhuizen, Path Integrals and Anomalies in Curved Space, (CUP, Cambridge, 2005).

30. A. Vilenkin and L.H. Ford, Phys. Rev. D 26, 1231 (1982); F. Scardigli, C. Gruber, P. Chen, Phys. Rev. D 83, 063507 (2011).

31. see, e.g., M. Tegmark et al., Phys. Rev. D 74, 123507 (2006).

32. Chibisov G V 1976 Soviet Physics Uspekhi 19 624; Lakes R 1998 Phys. Rev. Lett. 801826

33. H. Rumpf, Phys. Rev. D 33, 185 (1986); Prog. Theor. Phys. Suppl. 111, 63 (1993); T.C. de Aguiar, G. Menezes and N.F. Svaiter, Class. Quantum Grav. 26075003 (2009); J. Ambjorn, R. Loll, W. Westra and S. Zohren, Phys. Lett. B 680, 359 (2009). 